\title{
Influence of Sodium Chloride on the Formation and Dissociation Behavior of $\mathrm{CO}_{2}$ Gas Hydrates
}

\author{
Christine Holzammer, ${ }^{\dagger, \ddagger}$ Judith M. Schicks, ${ }^{\S}$ Stefan Will, ${ }^{\dagger, \ddagger}$ and Andreas S. Braeuer*, ${ }^{\dagger, \ddagger}$ \\ ${ }^{\dagger}$ Erlangen Graduate School in Advanced Optical Technologies (SAOT), Friedrich-Alexander-Universität Erlangen-Nürnberg (FAU), \\ Paul-Gordan-Str. 6, 91052 Erlangen, Germany \\ ${ }^{\ddagger}$ Lehrstuhl für Technische Thermodynamik (LTT), Friedrich-Alexander-Universität Erlangen-Nürnberg (FAU), Am Weichselgarten \\ 8, 91058 Erlangen, Germany \\ ${ }^{\S}$ Helmholtz Centre Potsdam, GFZ German Research Centre for Geosciences, Telegrafenberg, 14473 Potsdam, Germany
}

Supporting Information

\begin{abstract}
We present an experimental study on the formation and dissociation characteristics of carbon dioxide $\left(\mathrm{CO}_{2}\right)$ gas hydrates using Raman spectroscopy. The $\mathrm{CO}_{2}$ hydrates were formed from sodium chloride/water solutions with salinities of $0-$ $10 \mathrm{wt} \%$, which were pressurized with liquid $\mathrm{CO}_{2}$ in a stirred vessel at $6 \mathrm{MPa}$ and a subcooling of $9.5 \mathrm{~K}$. The formation of the $\mathrm{CO}_{2}$ hydrate resulted in a hydrate gel where the solid hydrate can be considered as the continuous phase that includes small amounts of a dispersed liquid water-rich phase that has not been converted to hydrate. During the hydrate formation process we quantified the fraction of solid hydrate, $x_{\mathrm{H}}$, and the fraction of the dispersed liquid water-rich phase, $x_{\mathrm{L}}$, from the signature of the hydroxyl $(\mathrm{OH})$ stretching vibration of the hydrate gel. We found that the fraction of hydrate $x_{\mathrm{H}}$ contained in the hydrate gel linearly depends on the salinity of the initial liquid water-rich phase. In addition, the ratio of $\mathrm{CO}_{2}$ and water was analyzed in the liquid water-rich phase before hydrate formation, in the hydrate gel during growth and dissociation, and after its complete dissociation again in the liquid water-rich phase. We observed a supersaturation of $\mathrm{CO}_{2}$ in the water-rich phase after complete dissociation of the hydrate gel and were able to show that the excess $\mathrm{CO}_{2}$ exists as dispersed micro- or nanoscale liquid droplets in the liquid water-rich phase. These residual nano- and microdroplets could be a possible explanation for the so-called memory effect.
\end{abstract}

\section{INTRODUCTION}

Gas hydrates are crystalline solids that consist of a hydrogenbonded, three-dimensional network of water molecules stabilized by included guest molecules. ${ }^{1,2}$ Especially in offshore drilling and gas pipelines the prevention of gas hydrates is of particular interest as high pressure and low temperature enhance gas hydrate formation. The undesired formation of hydrates can cause pipeline blockage or even destruction. ${ }^{3} \mathrm{~A}$ variety of substances that avert and/or delay their formation, called inhibitors, have been investigated. Thermodynamic inhibitors prevent the formation of gas hydrates by disturbing the hydrogen bond network in the water-rich phase before hydrate formation, shifting the hydrate forming equilibrium conditions to higher pressure and lower temperature. Raman spectroscopy is a powerful tool to analyze these disturbances because the hydroxyl $(\mathrm{OH})$-stretching vibration of water is sensitive to the development of hydrogen bonds. ${ }^{4,5}$ In a previous study we focused on the liquid water-rich phase before hydrate formation and analyzed the $\mathrm{OH}$-stretching vibration to quantify the effect of the salinity and the dissolved $\mathrm{CO}_{2}$ on the hydrogen bond network before hydrate formation. ${ }^{6}$ In contrast, here we focus on subsequent processes, which are the formation of the solid hydrate from the $\mathrm{CO}_{2}$ saturated liquid water-rich phase and the dissociation/melting of the hydrate phase as a function of the concentration of sodium chloride. The formation of the $\mathrm{CO}_{2}$ hydrate results in a hydrate gel where the solid hydrate can be considered as the continuous phase that includes a dispersed liquid water-rich phase that has not been converted to hydrate. Other authors ${ }^{7-10}$ quantified the fraction of liquid water included in the hydrate gel and the fraction of hydrate from the pressure drop in the isochoric vessel during hydrate formation. They applied the virial model for real gases and had to estimate the hydration number. The methodology that we present here allows for the determination of the fractions of solid hydrate and liquid water of the hydrate gel directly from the Raman signals of its $\mathrm{OH}$-stretching vibration. Therefore, it does not depend on an estimate of the hydration number.

In addition, we determined the ratio of carbon dioxide and water in the liquid water-rich phase before hydrate formation,

Received: June 2, 2017

Revised: August 11, 2017

Published: August 17, 2017 


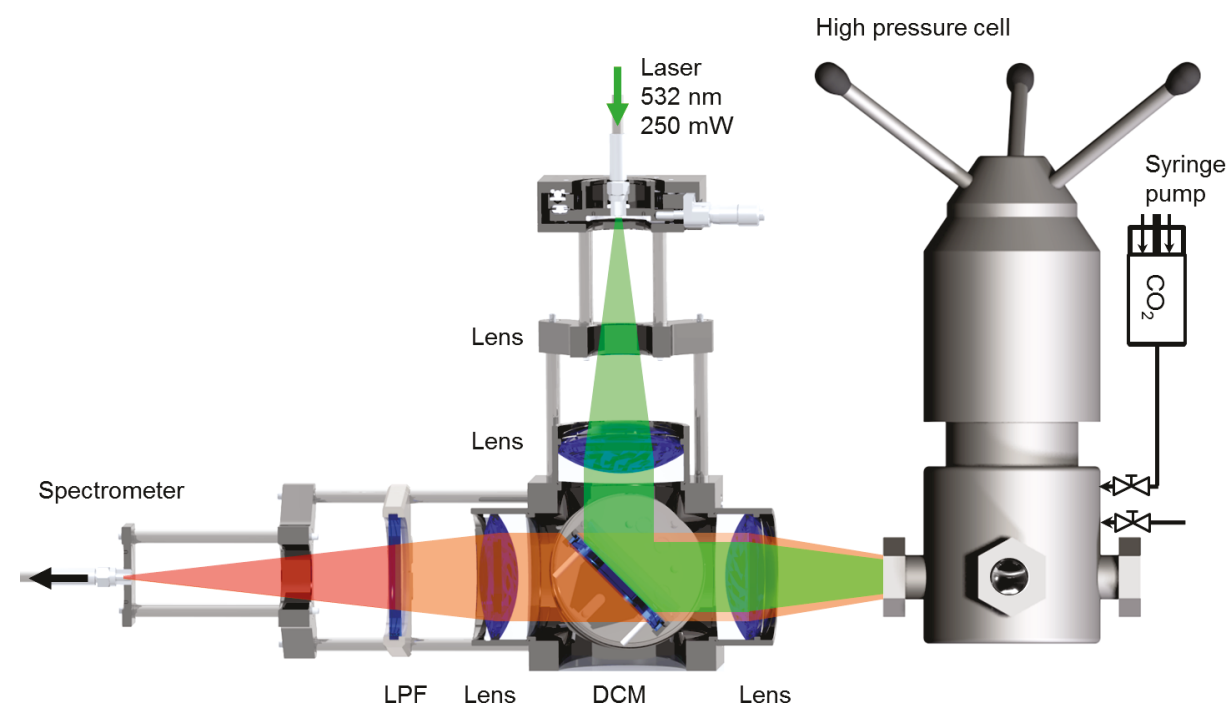

Figure 1. Sketch of the experimental setup including the high-pressure view cell and the Raman sensor head with beam paths of the laser excitation and the Raman signal.

during hydrate formation, during hydrate dissociation, and after the complete dissociation of the hydrate. Via these analyses we intended to investigate a phenomenon in the context of gas hydrates not yet fully understood, the so-called memory effect. It states that gas hydrates form easier from water that is gained from a molten gas hydrate than from a "freshly" prepared solution that has not experienced gas hydrate solution yet. The existence of this phenomenon is controversial, but three main reasons for it are given in the literature. ${ }^{11,12}$

The first explanation is the possible existence of residual structures in the liquid water-rich phase after gas hydrate melting. Some water molecules stay in a structure that is similar to the one of the gas hydrate cage. Neutron diffraction measurements indicate that a low concentration of hydrate crystals remains in the solution after the hydrate crystal appears to have melted and influences the local water structure. ${ }^{13}$ Consequently, a reformation of the hydrate cage is easier, and the hydrate formation appears faster. ${ }^{14}$

The second explanation is given by Zeng et al. ${ }^{15,16}$ According to them, the growth of a hydrate crystal causes an imprinting on the surface of impurities in the sample or the walls of the cell. The altered surface can act as a better nucleation site of hydrate, resulting in a facilitated hydrate nucleation.

The third explanation is that after the complete melting of the hydrate the remaining liquid water-rich phase contains excess hydrate guest molecules (here $\mathrm{CO}_{2}$ ), either dissolved in a supersaturated system or as dispersed micro bubbles/droplets in the form of a microemulsion. ${ }^{17}$ Recent studies have simulated the melting process of gas hydrates and found out that it results in a micro- and nanobubble (MNB) solution. Uchida et al. ${ }^{18,19}$ found evidence that the MNBs are stable for several hours. They detected the MNBs using a transmission electron microscope and investigated their influence on the memory effect. Bagherzadeh et al. ${ }^{20}$ performed molecular dynamic simulations on the decomposition of methane hydrate. They suggested that the MNBs may live long enough to be metastable and lead to the memory effect.

\section{MATERIALS AND METHODS}

The materials used in this work were deionized water with a conductivity less than $10 \mu \mathrm{S} / \mathrm{cm}$, sodium chloride (Alfa Aesar, optical grade), and carbon dioxide (Linde, molar purity 99.5\%). A schematic sketch of the experimental setup is given in Figure 1. It consists of two main parts: the high-pressure view cell and the optical setup.

High-Pressure Setup and Hydrate Formation Procedure. The high-pressure view cell, with a sample volume of 25 $\mathrm{mL}$, constructed by Separex, has four optical accesses. It has a syringe connection for filling and a screw fitting for emptying at the bottom. For pressurization with $\mathrm{CO}_{2}$, it is connected to a high-precision Teledyne ISCO syringe pump model 260-D. The pump head is cooled by a thermostat type F12-ED from Julabo to keep the $\mathrm{CO}_{2}$ inside the pump head liquid. The pressure inside the cell is measured with a pressure sensor type PTI-S-AG100-12AS, uncertainty 0.05 MPa, from Swagelok The temperature control of the cell is implemented by a cryo compact thermostat CF41 from Julabo, which circulates refrigerant through the double walled jacket of the cell. The temperature inside the cell is measured continuously by a PT100 thermocouple with a resolution of $0.05 \mathrm{~K}$ and an uncertainty of $0.12 \mathrm{~K}$ and recorded.

For the experiments, the cell is first flushed with $\mathrm{CO}_{2}$. After that, $20 \mathrm{~mL}$ of the water/salt-solution is filled into the cell using a syringe. The pressure is increased to $6 \mathrm{MPa}$ with the $\mathrm{CO}_{2}$ pump, and the temperature is set to the initial temperature, approximately $3 \mathrm{~K}$ above the equilibrium temperature of hydrate formation. The sample is continuously agitated with a magnetic stirrer. After $2 \mathrm{~h}$ at the initial set temperature and 6 $\mathrm{MPa}$, no further changes in the recorded Raman spectra are detectable. Therefore, the ratio of $\mathrm{CO}_{2}$ dissolved in the liquid water-rich phase $\left(\frac{n_{\mathrm{CO}_{2}}}{n_{\text {water }}}\right.$ ratio), extractable from the acquired Raman spectra, corresponds to the equilibrium ratio at the given pressure, temperature, and salinity. Afterward, the cell is cooled at $3 \mathrm{~K} / \mathrm{h}$ to the final set temperature that, as shown in Figure 2, is between the hydrate equilibrium temperature and the freezing temperature for the respective salinity. It is $9.5 \mathrm{~K}$ below the hydrate equilibrium temperature to achieve a high subcooling and approximately $1 \mathrm{~K}$ above the corresponding freezing temperature, which we estimated using Blagden's law ${ }^{21}$

$$
\Delta T=K_{\mathrm{f}} \cdot b
$$




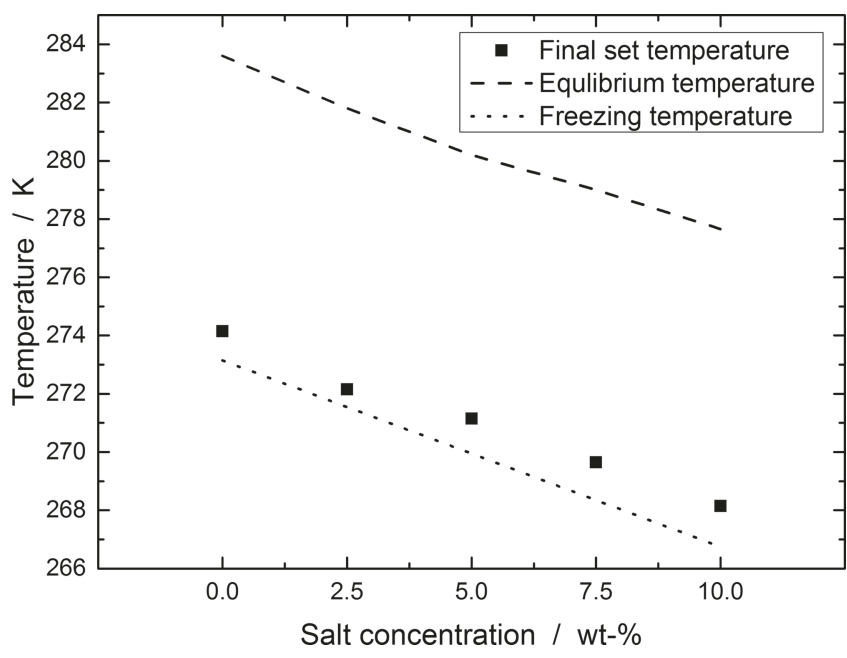

Figure 2. Sample concentrations with corresponding freezing temperatures, final set temperatures, and hydrate equilibrium temperatures. $^{22-26}$ The exact temperature values are listed in Table $S 1$ in the Supporting Information.

where $K_{\mathrm{f}}$ is the cryoscopic constant of water $\left(1.86 \mathrm{~K} \cdot \mathrm{kg} \cdot \mathrm{mol}^{-1}\right)$ and $b$ the molality.

After the final set temperature is reached, it takes between 1 and $7 \mathrm{~h}$ until spontaneous hydrate formation starts. Thirty min afterward, no changes in the Raman spectra acquired from the hydrate gel can be observed anymore, which signalizes stationary conditions. Subsequently, the sample is heated at 3 $\mathrm{K} / \mathrm{h}$ to the initial temperature, and the hydrate melts/ dissociates. The experiments were carried out three times for each salinity .

Raman Experimental Setup. The light source for the optical setup is a frequency-doubled Nd:YAG laser type 532250-AC from CNI that emits light with a wavelength of $532 \mathrm{~nm}$ and an output power of $250 \mathrm{~mW}$. The beam is widened and collimated by two lenses and guided by a dichroitic mirror (DCM) to a third lens. This lens, with a focal length of 100 $\mathrm{mm}$, focuses the beam into the high-pressure cell. The measuring volume has approximately a length of $5 \mathrm{~mm}$ (depth of field) and a diameter of $0.2 \mathrm{~mm}$. The detection of the inelastically scattered Raman signals is realized in a backscattering configuration. The dichroitic mirror reflects the elastically scattered green light, while light with a larger wavelength - the inelastically scattered Raman signal-is transmitted. Residual green light is withheld by a long pass filter (LPF), and the Raman signal is guided via a lens and a fiber into the spectrometer. Each Raman spectrum is acquired with a signal integration time of $1 \mathrm{~s}$. Raman spectra are recorded with a repetition rate of $1 \mathrm{~Hz}$. Each 10 consecutive Raman spectra are averaged, and the averaged spectra are correlated with a time-stamp and with the temperature measured inside the cell.

Hydrate Fraction Contained in the Hydrate Gel. The shape of the Raman signal of liquid water is known to be dependent on the development of the hydrogen bond network. ${ }^{4}$ Figure 3a shows area-normalized spectra of the $\mathrm{OH}$-stretching vibration of a liquid water-rich phase before hydrate formation $I_{\mathrm{L}}^{0}(\bar{\nu})$, the formed hydrate gel $I_{\mathrm{G}}^{0}(\bar{\nu})$ with liquid-water inclusions, and a pure hydrate phase $I_{\mathrm{H}}^{0}(\bar{\nu})$ without liquid-water inclusions. All spectra were recorded at $274 \mathrm{~K}$ and when the systems were pressurized with $\mathrm{CO}_{2}$. The spectrum of the pure hydrate phase was recorded with a Raman microscope from a single hydrate crystal according to the procedure described elsewhere, ${ }^{27}$ while the other spectra were recorded with the setup described above. There is a consensus in literature ${ }^{28,29}$ that the left shoulder of the band shape (2800$3350 \mathrm{~cm}^{-1}$ ) is attributable to strongly hydrogen-bonded water molecules, whereas the right shoulder $\left(3350-3800 \mathrm{~cm}^{-1}\right)$ is attributable to weakly hydrogen-bonded water molecules. Here "strong" and "weak" refer to the number of hydrogen bonds a water molecule forms with its neighboring water molecules but not to the strength of particular hydrogen bonds between neighboring water molecules. As the hydrate phase consists of a strongly hydrogen-bonded network, the intensity of the left shoulder of the pure hydrate spectrum exceeds that of the liquid water-rich phase before hydrate formation. Vice versa, in the liquid water-rich phase less hydrogen bonds are developed. Consequently, the right shoulder of the $\mathrm{OH}$-stretching vibration of the liquid water-rich phase is more pronounced than that of the pure hydrate. The hydrate gel, which consists of hydrate and an included liquid water-rich phase, gives a signal in between, because it is a superposition of both. Knowing that, it is possible to deconstruct the signal of the hydrate gel into its two original parts:
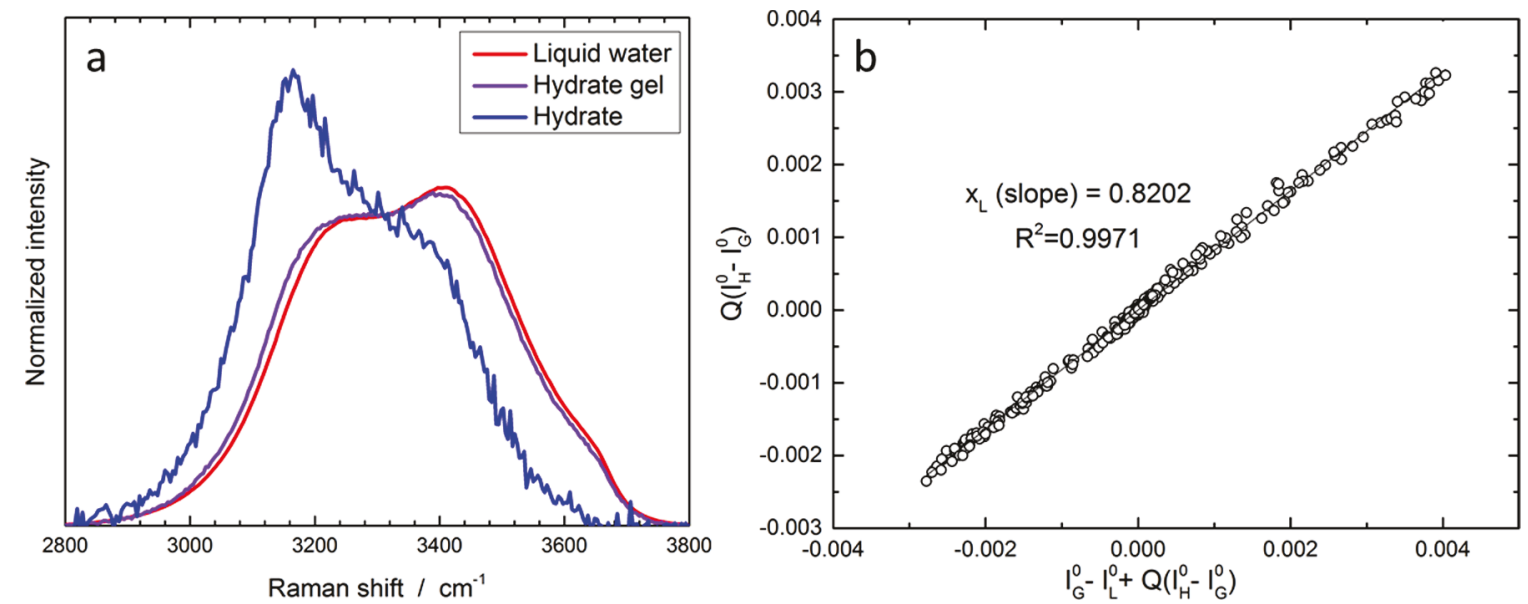

Figure 3. (a) Spectra of the $\mathrm{OH}$-stretching-vibration from the liquid water-rich phase, the hydrate gel, and the pure hydrate phase. (b) Best fit of eq 7 with the regression line for a water $/ \mathrm{CO}_{2}$ system at $\mathrm{T}=274 \mathrm{~K}$ and $p=6 \mathrm{MPa}$. 


$$
I_{\mathrm{G}}^{0}(\bar{\nu}) \cdot\left(\sigma_{\mathrm{L}} \cdot n_{\mathrm{L}}+\sigma_{\mathrm{H}} \cdot n_{\mathrm{H}}\right)=I_{\mathrm{L}}^{0}(\bar{\nu}) \cdot\left(\sigma_{\mathrm{L}} \cdot n_{\mathrm{L}}\right)+I_{\mathrm{H}}^{0}(\bar{\nu}) \cdot\left(\sigma_{\mathrm{H}} \cdot n_{\mathrm{H}}\right)
$$

$I_{\mathrm{i}}$ is the intensity of the Raman spectrum as a function of the Raman shift $\bar{\nu}$ of the compound i, in which the indices G, L, and $\mathrm{H}$ represent hydrate gel, the liquid water-rich phase, and the pure hydrate phase $\left(\mathrm{CO}_{2}\right.$ forms structure $\left.\mathrm{sI}\right)$, respectively. The superscript 0 denotes the area-normalized spectra. $\sigma_{\mathrm{i}}$ is the Raman scattering cross section of the compound $\mathrm{i}$, and $n_{\mathrm{i}}$ is the amount of substance of the compound i. $n_{\mathrm{L}}$ and $n_{\mathrm{H}}$ can be replaced by their molar fraction in the hydrate gel

$$
\begin{aligned}
& x_{\mathrm{L}}=\frac{n_{\mathrm{L}}}{n_{\mathrm{L}}+n_{\mathrm{H}}}=\frac{n_{\mathrm{L}}}{n_{\mathrm{G}}} \\
& x_{\mathrm{H}}=\frac{n_{\mathrm{H}}}{n_{\mathrm{L}}+n_{\mathrm{H}}}=\frac{n_{\mathrm{H}}}{n_{\mathrm{G}}}
\end{aligned}
$$

which must sum to 1 :

$$
x_{\mathrm{L}}=1-x_{\mathrm{H}}
$$

We now introduce $Q$ as the ratio of the Raman scattering cross sections of water in its hydrate phase and its liquid phase, ${ }^{30}$ respectively

$$
Q=\frac{\sigma_{\mathrm{H}}}{\sigma_{\mathrm{L}}}
$$

which we, because of the similarity of the Raman spectra of ice and hydrate sI, approximate to $Q=0.607$ by the ratio of the Raman scattering cross sections of water in its ice phase and in its liquid phase. ${ }^{27}$ Inserting $x_{\mathrm{L}}$ and $Q$ in eq 2 yields the linear relation

$$
\begin{aligned}
& Q\left[I_{\mathrm{H}}^{0}(\bar{\nu})-I_{\mathrm{G}}^{0}(\bar{\nu})\right] \\
& \quad=x_{\mathrm{L}}\left[\left(I_{\mathrm{G}}^{0}(\bar{\nu})-I_{\mathrm{L}}^{0}(\bar{\nu})\right)+Q\left(I_{\mathrm{H}}^{0}(\bar{\nu})-I_{\mathrm{G}}^{0}(\bar{\nu})\right)\right]
\end{aligned}
$$

$I_{\mathrm{H}}^{0}(\bar{\nu}), I_{\mathrm{L}}^{0}(\bar{\nu})$, and $I_{\mathrm{G}}^{0}(\bar{\nu})$ are the recorded spectra shown in Figure 3a (see the description above). By evaluating eq 7 for Raman shifts from 2800 to $3800 \mathrm{~cm}^{-1}$, which is visualized in Figure $3 b, x_{L}$ can be derived from the slope in Figure $3 b$ with a linear least-squares regression. For this method we assume the ratio of the Raman cross sections $Q$ to be constant for the Raman shifts evaluated.

To characterize the quality of the fit, we used the coefficient of determination $R^{2}$.

With this method it is possible to determine the molar fraction of solid hydrate $x_{\mathrm{H}}=1-x_{\mathrm{L}}$ and the included liquid water-rich dispersed phase $x_{\mathrm{L}}$ in a hydrate gel and investigate the influence of different inhibitors on this property.

Determination of the $\mathrm{CO}_{2}$ /Water Ratio. The intensity (integrated peak area) $I_{\mathrm{i}}$ of the Raman peak for species $\mathrm{i}$ is dependent on the irradiance on the sample, $i_{0}$; the instrumental efficiency of the optical and electronic response, $\eta_{i}$; the Raman scattering cross section, $\sigma_{\mathrm{i}}$; and the amount of substance in the irradiated volume, $n_{\mathrm{i}}$. ${ }^{31,32}$ Therefore, the intensity ratio of the Raman signals of $\mathrm{CO}_{2}\left(\mathrm{I}_{\mathrm{CO}_{2}}\right)$ and water $\left(I_{\text {water }}\right)$

$$
\frac{I_{\mathrm{CO}_{2}}}{I_{\text {water }}}=\frac{i_{0} \cdot \eta_{\mathrm{CO}_{2}} \cdot \sigma_{\mathrm{CO}_{2}}}{i_{0} \cdot \eta_{\text {water }} \cdot \sigma_{\text {water }}} \frac{n_{\mathrm{CO}_{2}}}{n_{\text {water }}}=a \frac{n_{\mathrm{CO}_{2}}}{n_{\text {water }}}
$$

is proportional to the molar ratio of $\mathrm{CO}_{2}$ and water in the sample. For systems in which $i_{0}, \eta_{\mathrm{i}}$, and $\sigma_{\mathrm{i}}$ are constant, these parameters can be combined to the proportionality constant $a$. Here, $I_{\mathrm{CO}_{2}}$ is the integral of the two bands around 1280 and
$1380 \mathrm{~cm}^{-1}$ (see Table 1), attributed to the lower $\left(\nu_{1}\right)$ and upper $\left(\nu_{\mathrm{u}}\right)$ band of the Fermi dyad of $\mathrm{CO}_{2}$. These bands arise from

Table 1. Raman Shifts of the Upper $\left(v_{\mathrm{u}}\right)$ and Lower $\left(v_{1}\right)$ Band of the $\mathrm{CO}_{2}$ Fermi Dyad for Different States of $\mathrm{CO}_{2}{ }^{38,39}$

\begin{tabular}{lcc}
\multicolumn{1}{c}{ state $\mathrm{CO}_{2}$} & $\begin{array}{c}\text { positions } \mathrm{CO}_{2} v_{1} \\
\left(\mathrm{~cm}^{-1}\right)\end{array}$ & $\begin{array}{c}\text { positions } \mathrm{CO}_{2} v_{\mathrm{u}} \\
\left(\mathrm{cm}^{-1}\right)\end{array}$ \\
gaseous $\mathrm{CO}_{2}$ & 1285 & 1388 \\
liquid $\mathrm{CO}_{2}$ & 1280 & 1386 \\
$\mathrm{sI} \mathrm{CO}_{2}$ hydrate & 1276 & 1381 \\
$\mathrm{CO}_{2}$ dissolved in liquid \\
water
\end{tabular}

Fermi resonance of the $\nu_{1}$ symmetric stretching $\mathrm{O}=\mathrm{C}=\mathrm{O}$ and $2 \nu_{2}$ bending overtone of the $\mathrm{CO}_{2}$ molecules. ${ }^{33,34} I_{\text {water }}$ is the integral of the Raman signal of the $\mathrm{OH}$-vibration between 2800 and $3800 \mathrm{~cm}^{-1}$ (see Figure $5 \mathrm{~b}$ ). The proportionality constant $a$ $=2.76$ is determined for the liquid water-rich phase by measuring the intensity ratio $\frac{I_{\mathrm{CO}_{2}}}{I_{\text {water }}}$ at pressures and temperatures, for which the saturation molar ratio $\frac{n_{\mathrm{CO}_{2}}}{n_{\text {water }}}$ is known from literature. ${ }^{35-37}$ The saturation values used for calibration are given in Table S2. The Raman scattering cross section of liquid water differs from that of water in the hydrate structure. Therefore, the proportionality constant $a$ that we found for the liquid water-rich phase is not applicable to determine the ratio $\frac{n_{\mathrm{CO}_{2}}}{n_{\text {water }}}$ in the pure hydrate phase nor in the hydrate gel.

Determination of the Raman Shift of the Two Main Peaks of the $\mathrm{CO}_{2}$ Fermi Dyad. The Raman shifts of the upper $\left(v_{\mathrm{u}}\right)$ and lower band $\left(v_{1}\right)$ of the $\mathrm{CO}_{2}$ Fermi dyad depend on the chemical environment of the $\mathrm{CO}_{2}$ molecules. The Raman shifts of $v_{\mathrm{u}}$ and $v_{1}$ are therefore different for $\mathrm{CO}_{2}$ in the gas phase, for $\mathrm{CO}_{2}$ dissolved in a water-rich phase, for $\mathrm{CO}_{2}$ incorporated as guest molecule in a hydrate cage, or for $\mathrm{CO}_{2}$ present in a $\mathrm{CO}_{2}$-rich environment, e.g., in the form of $\mathrm{CO}_{2}$ droplets. $^{38}$ The Raman shifts are also weakly temperaturedependent, but this can be neglected in the temperature range investigated. Table 1 shows the Raman shifts of the upper and lower band of the $\mathrm{CO}_{2}$ Fermi dyad for different states of carbon dioxide.

In order to determine the Raman shift of the upper and lower band of the $\mathrm{CO}_{2}$ Fermi dyad, we fitted pseudo-Voigt profiles to each of the bands that are shown in Figure 5b. The center of the pseudo-Voigt profiles, peak width, intensity, and shape were fitted as described previously. ${ }^{40}$

\section{RESULTS AND DISCUSSION}

The following discussion of the characteristics of one measurement cycle will be performed for systems without salt exemplarily. Measurement cycles for systems containing $\mathrm{NaCl}$ look very similar and are shown in the Supporting Information.

Figure 4 shows photographs of the water $/ \mathrm{CO}_{2}$ sample acquired through the windows of the high-pressure cell during one measurement cycle.

Figure $5 \mathrm{~b}$ shows typical Raman spectra acquired from the liquid water-rich phase before hydrate formation [states 1 (red) and 2 (orange)], from the hydrate gel [state 3 (blue)] and from the liquid water-rich phase after complete dissociation of the hydrate [state 6 (green)]. From the Raman spectra the evolution of the $\frac{I_{\mathrm{CO}_{2}}}{I_{\text {water }}}$ ratio is extracted and shown as a function 


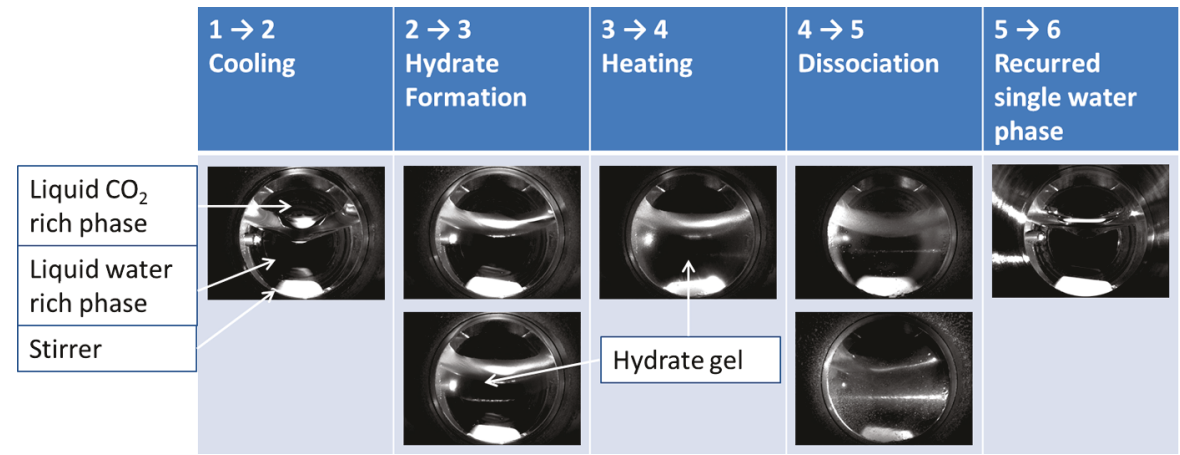

Figure 4. Photographs of the water/ $\mathrm{CO}_{2}$ sample acquired through the windows of the high-pressure cell during one measurement cycle. The laser beam can be seen weakly in the photographs corresponding to the state changes $2 \rightarrow 3,3 \rightarrow 4$, and $4 \rightarrow 5$. For the two state changes $2 \rightarrow 3$ and $4 \rightarrow$ 5 , two photographs are shown. The lower one corresponds to a later instant of time.
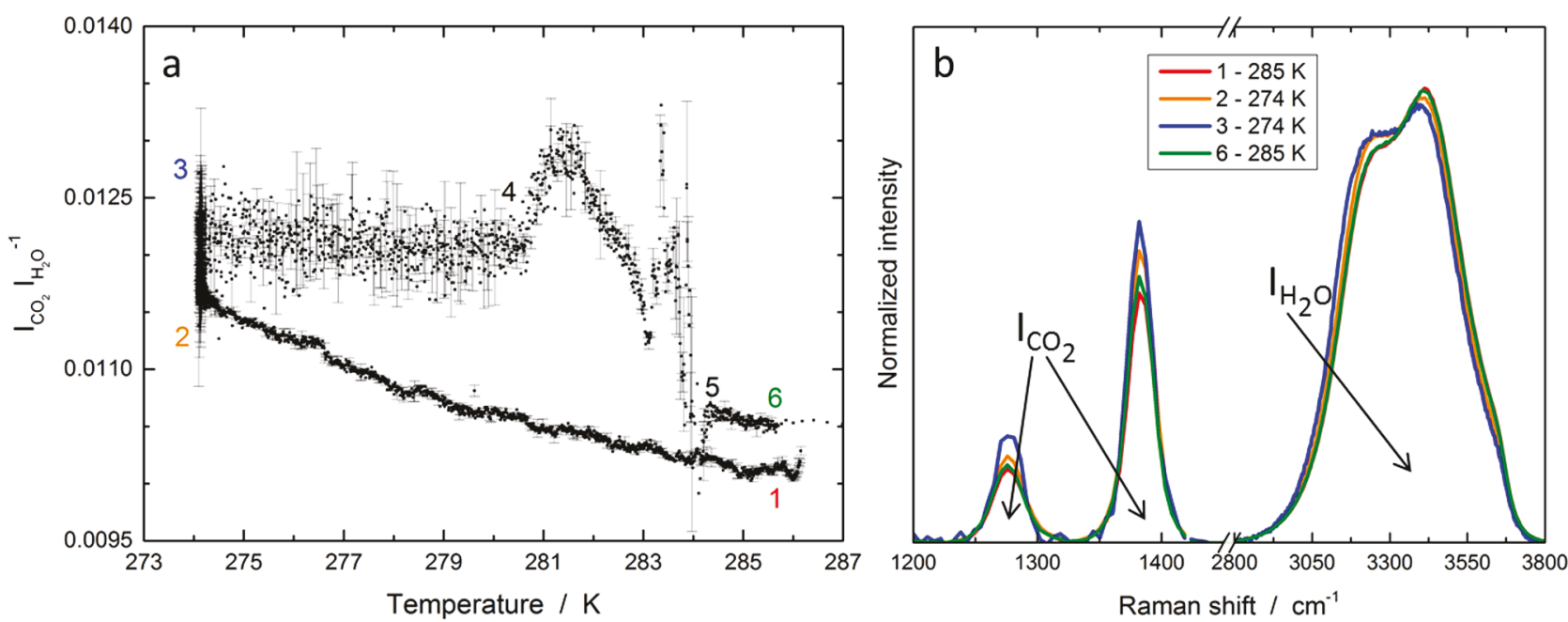

Figure 5. (a) Evolution of the $\frac{I_{\mathrm{CO}_{2}}}{I_{\text {water }}}$ ratio in the liquid water-rich phase $(1 \rightarrow 2$ and $5 \rightarrow 6)$ and in the hydrate gel $(3 \rightarrow 4)$ for a water/CO system. (b) Example Raman spectra from the liquid water-rich phase and from the gel corresponding to the state numbers 1, 2, 3, and 6 labeled in panel a.

of the temperature during an entire measurement cycle. The numbers $1-6$ provided in Figure 5a,b correspond to identical states also shown in Figure 4. One measurement cycle can be divided into five main parts.

$1 \rightarrow 2$ : Cooling of the Liquid Water-Rich Phase. From 1 $\rightarrow 2$ the sample, which consists of liquid water and is saturated with $\mathrm{CO}_{2}$ at $6 \mathrm{MPa}$, is continuously cooled from 286 to $274 \mathrm{~K}$. In this period, the intensity of the Raman signal from 2800 to $3350 \mathrm{~cm}^{-1}$ attributable to strongly hydrogen-bonded water molecules increases, whereas the intensity of the Raman signal from 3350 to $3800 \mathrm{~cm}^{-1}$ assigned to weakly hydrogen-bonded water molecules decreases. As the kinetic energy, or mobility, respectively, decreases with decreasing temperature, more water molecules get strongly hydrogen-bonded. The $\frac{I_{\mathrm{CO}_{2}}}{I_{\text {water }}}$ ratio increases linearly as the solubility of $\mathrm{CO}_{2}$ increases with decreasing temperature.

$2 \rightarrow$ 3: Hydrate Formation. At the temperature of $274 \mathrm{~K}$ (final set temperature), it takes $1-7 \mathrm{~h}$ until rapid hydrate growth appears, caused by the stochastic characteristics of nucleation: usually beginning at the interphase of the liquid water-rich and the $\mathrm{CO}_{2}$-rich phase, the hydrate phase spreads downward until the stirrer is blocked and stops to rotate. Though the sample appears completely solid, not all the water participates in the hydrate phase, but portions remain as liquid water-rich inclusions. Therefore, the $\mathrm{OH}$-stretching vibration gained from the hydrate gel is a mix of the solid hydrate phase and the liquid water-rich phase. The formation of the hydrate gel is accompanied by a sudden increase of the $\frac{I_{\mathrm{CO}_{2}}}{I_{\text {water }}}$ ratio (from 0.0115 to 0.0125 ) and a further increase of the Raman intensity from 2800 to $3350 \mathrm{~cm}^{-1}$ of the $\mathrm{OH}$-stretching vibration. This was expected, as more water molecules are hydrogen-bonded and also more carbon dioxide can be accumulated in the hydrate phase than in the coexisting liquid water-rich phase. ${ }^{3}$

$3 \rightarrow 4$ : Heating of the System. From $3 \rightarrow 4$ the hydrate gel is heated. The $\frac{I_{\mathrm{CO}_{2}}}{I_{\text {water }}}$ ratio stays at a fixed value, but the intensity of the $\mathrm{OH}$-stretching vibration from 2800 to 3350 $\mathrm{cm}^{-1}$ decreases, whereas from 3350 to $3800 \mathrm{~cm}^{-1}$ it increases (not shown in Figure 5). The stationary $\frac{I_{\mathrm{CO}_{2}}}{I_{\text {water }}}$ ratio indicates that no further significant amount of $\mathrm{CO}_{2}$ gets into the hydrate gel, most likely because the $\mathrm{CO}_{2}$ transport is limited because of the slow diffusion processes through the gel. The constant amount of $\mathrm{CO}_{2}$ in the hydrate gel can be considered as an indicator for the stop of formation of additional hydrate. The change in the $\mathrm{OH}$-stretching vibration can be explained by the fact that the hydrate gel consists of a liquid water-rich phase and hydrate phase. The $\mathrm{OH}$-stretching vibration of the hydrate phase is, in the temperature interval investigated, not temperature sensitive 
(see Figure S3), but the $\mathrm{OH}$-stretching vibration of the included liquid water-rich phase in the hydrate gel is temperature sensitive. Consequently, the $\mathrm{OH}$-stretching vibration of the hydrate gel changes with temperature, while the $\frac{I_{\mathrm{CO}_{2}}}{I_{\text {water }}}$ ratio remains unchanged.

$4 \rightarrow$ 5: Dissociation/Melting of the Hydrate Phase. At point 4 the hydrate starts to dissociate. The first indication for this effect is that the magnetic stirrer starts to move again. At this time the hydrate gel still exists as an interphase below the upper $\mathrm{CO}_{2}$-rich phase. We suppose that the initial increase of the $\frac{I_{\mathrm{CO}_{2}}}{I_{\text {water }}}$ ratio is an effect caused by a temporary local increase of the $\mathrm{CO}_{2}$ concentration within the measurement volume. When $\mathrm{CO}_{2}$ is released from the hydrate cages, it agglomerates to droplets (bigger than micro-nanoscale). While rising to the upper $\mathrm{CO}_{2}$ rich phase, they cross the measuring volume and cause an increase of the $\frac{I_{\mathrm{CO}_{2}}}{I_{\text {water }}}$ ratio. With further increase of the temperature, more and more hydrate dissociates, resulting in complete melting of the hydrate phase. Once the $\mathrm{CO}_{2}$ droplets have coagulated and separated from the liquid water-rich phase, the $\frac{I_{\mathrm{CO}_{2}}}{I_{\text {water }}}$ ratio drops toward state 5 .

$5 \rightarrow$ 6: Recurred Water Phase. At point 5 the hydrate is completely dissociated and all visible $\mathrm{CO}_{2}$ droplets are gone from the liquid water-rich phase. Visually the water-rich phase between states 5 and 6 is as transparent as the original liquid water-rich phase between states 1 and 2 . The shape of the $\mathrm{OH}$ stretching vibration from $5 \rightarrow 6$ is the same as for the cooling period $1 \rightarrow 2$ at the same temperature (cf. Raman spectra 1 and 6 in Figure $5 b)$. However, the $\frac{I_{\mathrm{CO}_{2}}}{I_{\text {water }}}$ ratio for a given temperature is higher than at the same temperature for the cooling period, although there are no visible $\mathrm{CO}_{2}$ droplets in the water-rich phase.

Evolutions of the $\frac{I_{\mathrm{CO}_{2}}}{I_{\text {water }}}$ ratio for different salt concentrations that we show in the Supporting Information differ in the absolute amount of the $\frac{I_{\mathrm{CO}_{2}}}{I_{\text {water }}}$ ratio, as the addition of salt reduces the solubility of nonpolar gases in water. ${ }^{6}$ Nevertheless the characteristics of the measurement cycles are identical.

Figure 6 shows the saturation $S=\frac{\frac{n_{\mathrm{CO}}}{n_{\text {water }}}}{\left(\frac{n_{\mathrm{CO}}}{n_{\text {water }}}\right)^{*}}=\frac{a}{a} \frac{\frac{I_{\mathrm{CO}_{2}}}{I_{\text {water }}}}{\left(\frac{I_{\mathrm{CO}}}{I_{\text {water }}}\right)^{*}}$ in the recurred liquid water-rich phase for different salinities.

The $\frac{I_{\mathrm{CO}_{2}}}{I_{\text {water }}}$ ratio that we measured during cooling $(1 \rightarrow 2)$ at the respective temperature and salt concentration corresponds to the equilibrium $\left(\frac{I_{\mathrm{CO}_{2}}}{I_{\text {water }}}\right)^{*}$ ratio. With values around $2-3 \%$, the supersaturation in the recurred water phase $(5 \rightarrow 6)$ is significant. It can also be seen that the supersaturation is not a function of the salinity. It stays at a value of about $3 \%$ for all salinities.

To shed further light on the origin of the supersaturation of the recurred liquid water-rich phase, we also investigated the position of the $\mathrm{CO}_{2}$ Fermi dyad during the whole measurement cycle. Figure 7 shows the position of the Raman shifts of the upper and lower band of the Fermi dyad $\nu_{\mathrm{u}}$ and $\nu_{1}$ of the $\mathrm{CO}_{2}$ measured in the liquid water-rich phase as a function of temperature representing one measurement cycle.

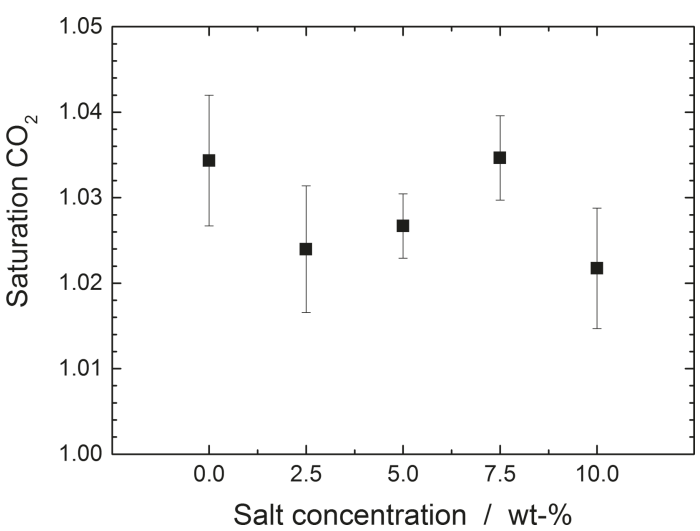

Figure 6. Saturation of the recurred water phase of carbon dioxide for different salt concentrations.

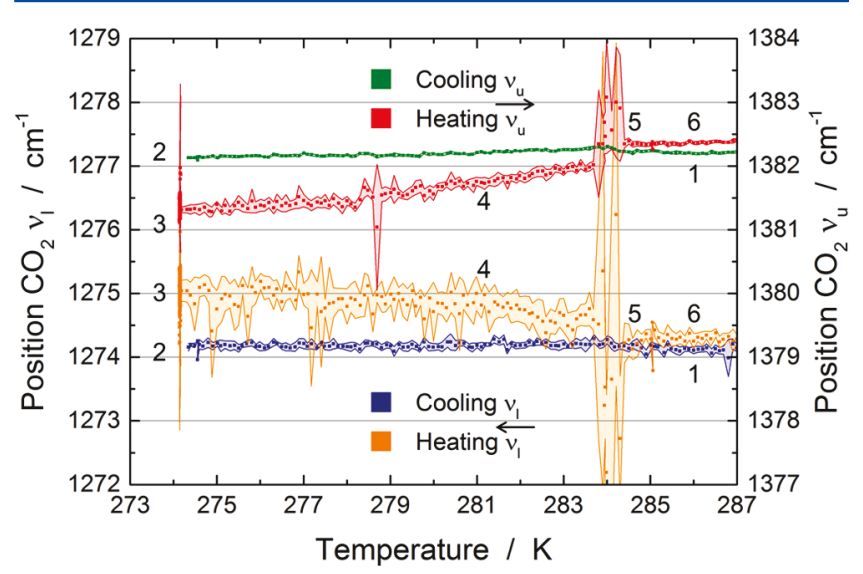

Figure 7. Positions of the upper $\left(v_{\mathrm{u}}\right)$ and lower $\left(v_{1}\right)$ band of the $\mathrm{CO}_{2}$ Fermi dyad during one measurement cycle.

The shadowed area corresponds to the double standard deviation of the measurement points, and the state numbers 16 correspond to those used in Figure 5. The values of both peaks remain at a constant value for the cooling process and are in good agreement with values reported in the literature for $\mathrm{CO}_{2}$ dissolved in water. ${ }^{38}$ With hydrate formation the lower band $\left(v_{1}\right)$ shifts from 1274 to $1275 \mathrm{~cm}^{-1}$, whereas the upper band $\left(v_{\mathrm{u}}\right)$ shifts from 1382 to $1381 \mathrm{~cm}^{-1}$. Keeping in mind that the hydrate gel does not consist of pure hydrate, meaning that not all $\mathrm{CO}_{2}$ molecules are incorporated in hydrate cages, but are also dissolved in the included liquid water-rich phase, the measured Raman shifts from the hydrate gel cannot reach the Raman shifts reported for pure hydrate, but only shift toward these values. The Raman shifts of the $\mathrm{CO}_{2}$ Fermi dyad stay constant at the beginning of heating. With dissociation of the hydrate phase the Raman shifts move toward those of $\mathrm{CO}_{2}$ dissolved in water. After complete melting of the hydrate, the positions of the two main peaks of the $\mathrm{CO}_{2}$ Fermi dyad are slightly higher than the Raman shifts we measured for $\mathrm{CO}_{2}$ dissolved in the liquid water-rich phase during the cooling period $(1 \rightarrow 2)$. This can be explained by the existence of liquid $\mathrm{CO}_{2}$-rich micro- and nanodroplets. Because the amount of $\mathrm{CO}_{2}$ contained inside the microdroplets is small, the shift toward the position of liquid $\mathrm{CO}_{2}$ is also small. Overall, this finding corroborates the hypothesis that $\mathrm{CO}_{2}$ micro- and nanodroplets remain in the aqueous phase after the dissociation of the hydrate phase. According to Uchida et al. ${ }^{18,19}$ the memory effect is caused by an excess of guest molecules in the aqueous 
phase, namely that MNBs may explain the facilitated formation of gas hydrates in a solution that has already experienced gas hydrate formation before.

Figure 8 shows the molar fraction of pure hydrate $x_{\mathrm{H}}$ contained in the hydrate gel at the end of the hydrate

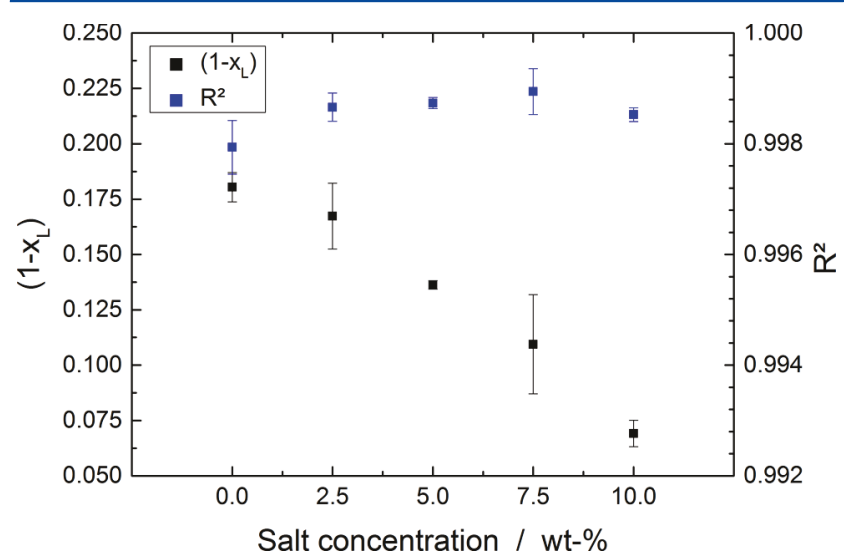

Figure 8. Molar fraction of hydrate contained in the hydrate gel for different salt concentrations.

formation (state 3) for different salinities. The temporal evolutions of $x_{\mathrm{H}}(t)$ during the formation of the hydrate gel $(2 \rightarrow 3)$ are shown for various salinities in the Supporting Information. The maximal fraction of hydrate in a gel formed from the liquid water-rich phase that did not contain any salt. From this maximum of $0.175, x_{\mathrm{H}}$ reduces monotonically with increasing salinity until it reaches a value of 0.075 for the highest analyzed content of $\mathrm{NaCl}$ of $10 \%$. The coefficient of determination $R^{2}$ of the linear regression (compare Figure $3 \mathrm{~b}$ ) is very good for all experiments with values above 0.997 .

There are two possible aspects causing the diminution of the molar fraction of hydrate in the gel. Water molecules are needed for the dissolution of the $\mathrm{Na}^{+}$and $\mathrm{Cl}^{-}$ions and build a hydration shell around the ions inducing subsequent effects:

(1) As these molecules are not available for the solution of $\mathrm{CO}_{2}$, the solubility of $\mathrm{CO}_{2}$ in the water-rich phase decreases. The incorporated guest molecules in the hydrogen-bonded water structure in a gas hydrate stabilize the gas hydrate crystal. As fewer $\mathrm{CO}_{2}$ molecules are available for the hydrate formation, fewer water molecules can be stabilized in a gas hydrate phase with the result that more liquid water is incorporated in the hydrate gel.

(2) As these molecules are also not available for the development of hydrogen bonds with other water molecules the addition of salt also leads to a weakening of the hydrogen bond network in the liquid water-rich phase. As the hydrogen bond network is weaker, the more salt is dissolved in the liquid water-rich phase, the formation of a strongly hydrogen-bonded hydrate network is harder to establish.

\section{CONCLUSION}

In this work we presented a method to determine all at once from the acquired Raman spectra only (i) the molar fraction of hydrate contained in the hydrate gel, (ii) the ratio of $\mathrm{CO}_{2} /$ water, and (iii) the states in which $\mathrm{CO}_{2}$ exists in the hydrate forming systems. The method was applied to investigate the influence of sodium chloride on the formation of carbon dioxide hydrate. We showed that the addition of a thermodynamic inhibitor not only changes the equilibrium conditions of the hydrate formation but also diminishes the amount of solid hydrate formed. We attributed this effect mainly to the reduced amount of dissolved carbon dioxide in the water-rich phase with increasing salinity. With the method presented here other inhibitors, both thermodynamic and kinetic, can be characterized with regard to their inhibition effect, meaning their influence on the conversion from liquid water to hydrate.

Additionally we were able to detect an excess amount of carbon dioxide in the water-rich phase after complete dissociation of the hydrate. By determining the position of the carbon dioxide dyad we provided evidence for the development of a micro- and nanodroplet emulsion from the dissociation of gas hydrates, which may, at least partially, explain the memory effect.

Basically, it is possible to apply these techniques to other types of gas hydrates, if the guest molecule is Raman active. However, it is possible that a Raman sensor with a higher sensitivity and/or a higher resolution is needed, as the solubilities of other guest molecules, like methane or ethane, are considerably lower than the solubility of carbon dioxide.

\section{ASSOCIATED CONTENT}

\section{Supporting Information}

The Supporting Information is available free of charge on the ACS Publications website at DOI: 10.1021/acs.jpcb.7b05411.

Evolution of the molar fraction of hydrate during hydrate formation and the intensity ratio $\mathrm{CO}_{2}$ /water during one measurement cycle each for different salinities; $\mathrm{OH}$ stretching vibration of the pure hydrate phase for different temperatures and a table with the freezing-, equilibrium- and set temperatures for different salinities; solubility values of carbon dioxide in water used for the proportionality constant $a$ (PDF)

\section{AUTHOR INFORMATION}

\section{Corresponding Author}

*E-mail: Andreas.Braeuer@fau.de. Phone: (+49)9131-8525853. Fax: (+49)9131-85-25851.

ORCID ${ }^{\circ}$

Christine Holzammer: 0000-0001-8163-2840

\section{Notes}

The authors declare no competing financial interest.

\section{ACKNOWLEDGMENTS}

The project leading to this result has received funding from the European Union's Horizon 2020 research and innovation programme under Grant Agreement No. 637654 (Inhomogeneities). Furthermore, we gratefully acknowledge the funding of the Erlangen Graduate School in Advanced Optical Technologies (SAOT) by the German Research Foundation (DFG) in the framework of the German excellence initiative.

\section{REFERENCES}

(1) Sloan, E. D. Fundamental Principles and Applications of Natural Gas Hydrates. Nature 2003, 426 (6964), 353-363.

(2) Von Stackelberg, M.; Gotzen, O.; Pietuchovsky, J.; Wirtscher, O.; Fruhbuss, H.; Meinhold, W. Struktur und Formel der Gashydrate. Fortschr. Mineral. 1947, 26, 122-124.

(3) Sloan, E. D.; Koh, C. Clathrate Hydrates of Natural Gases, 3rd ed.; CRC Press: Boca Raton, FL, 2007. 
(4) Bassing, D.; Braeuer, A. S. The Lag Between Micro-and MacroMixing in Compressed Fluid Flows. Chem. Eng. Sci. 2017, 163, 105113.

(5) Schuster, J. J.; Siegler, P.; Guenther, A.; Wirth, K.-E.; Braeuer, A. S. Simultaneous Analysis of the Dispersed Liquid and the Bulk Gas Phase of Water Sprays Using Raman Spectroscopy. Appl. Spectrosc. 2016, 70, 1055.

(6) Holzammer, C.; Finckenstein, A.; Will, S.; Braeuer, A. S. How Sodium Chloride Salt Inhibits the Formation of $\mathrm{CO}_{2}$ Gas Hydrates. J. Phys. Chem. B 2016, 120 (9), 2452-2459.

(7) Linga, P.; Daraboina, N.; Ripmeester, J. A.; Englezos, P. Enhanced Rate of Gas Hydrate Formation in a Fixed Bed Column Filled With Sand Compared to a Stirred Vessel. Chem. Eng. Sci. 2012, 68 (1), 617-623.

(8) Farhang, F.; Nguyen, A. V.; Hampton, M. A. Influence of Sodium Halides on the Kinetics of $\mathrm{CO}_{2}$ Hydrate Formation. Energy Fuels 2014, 28 (2), 1220-1229.

(9) Mekala, P.; Babu, P.; Sangwai, J. S.; Linga, P. Formation and Dissociation Kinetics of Methane Hydrates in Seawater and Silica Sand. Energy Fuels 2014, 28 (4), 2708-2716.

(10) Yang, S. H. B.; Babu, P.; Chua, S. F. S.; Linga, P. Carbon Dioxide Hydrate Kinetics in Porous Media With and Without Salts. Appl. Energy 2016, 162, 1131-1140.

(11) Ripmeester, J. A.; Alavi, S. Some Current Challenges in Clathrate Hydrate Science: Nucleation, Decomposition and the Memory Effect. Curr. Opin. Solid State Mater. Sci. 2016, 20 (6), 344-351.

(12) Sowa, B.; Maeda, N. Statistical Study of the Memory Effect in Model Natural Gas Hydrate Systems. J. Phys. Chem. A 2015, 119 (44), 10784-10790.

(13) Thompson, H.; Soper, A. K.; Buchanan, P.; Aldiwan, N.; Creek, J. L.; Koh, C. A. Methane Hydrate Formation and Decomposition: Structural Studies Via Neutron Diffraction and Empirical Potential Structure Refinement. J. Chem. Phys. 2006, 124 (16), 164508.

(14) Sloan, E. D.; Subramanian, S.; Matthews, P.; Lederhos, J.; Khokhar, A. Quantifying Hydrate Formation and Kinetic Inhibition. Ind. Eng. Chem. Res. 1998, 37 (8), 3124-3132.

(15) Zeng, H.; Moudrakovski, I. L.; Ripmeester, J. A.; Walker, V. K. Effect of Antifreeze Protein on Nucleation, Growth and Memory of Gas Hydrates. AIChE J. 2006, 52 (9), 3304-3309.

(16) Zeng, H.; Wilson, L. D.; Walker, V. K.; Ripmeester, J. A. Effect of Antifreeze Proteins on the Nucleation, Growth, and the Memory Effect During Tetrahydrofuran Clathrate Hydrate Formation. J. Am. Chem. Soc. 2006, 128 (9), 2844-2850.

(17) Rodger, P. Methane Hydrate: Melting and Memory. Ann. N. Y. Acad. Sci. 2000, 912 (1), 474-482.

(18) Uchida, T.; Yamazaki, K.; Gohara, K. Generation of Micro-and Nano-Bubbles in Water by Dissociation of Gas Hydrates. Korean J. Chem. Eng. 2016, 33 (5), 1749-1755.

(19) Uchida, T.; Yamazaki, K.; Gohara, K. Gas Nanobubbles as Nucleation Acceleration in the Gas-Hydrate Memory Effect. J. Phys. Chem. C 2016, 120 (47), 26620-26629.

(20) Bagherzadeh, S. A.; Alavi, S.; Ripmeester, J.; Englezos, P. Formation of Methane Nano-Bubbles During Hydrate Decomposition and their Effect on Hydrate Growth. J. Chem. Phys. 2015, 142 (21), 214701.

(21) Atkins, P.; de Paula, J. Atkins' Physical Chemistry; OUP: Oxford, 2014.

(22) Dholabhai, P. D.; Kalogerakis, N.; Bishnoi, P. R. Equilibrium Conditions for Carbon Dioxide Hydrate Formation in Aqueous Electrolyte Solutions. J. Chem. Eng. Data 1993, 38 (4), 650-654.

(23) Larson, S. D. Phase Studies of the Two-Component Carbon Dioxide-Water System: Involving the Carbon Dioxide Hydrate. University of Illinois, 1955.

(24) Lemmon, E. W.; McLinden, M. O.; Friend, D. G. NIST Chemistry WebBook, 2015; NIST Standard Reference Database Number 69.

(25) Ng, H.-J.; Robinson, D. B. Hydrate Formation in Systems Containing Methane, Ethane, Propane, Carbon Dioxide or Hydrogen
Sulfide in the Presence of Methanol. Fluid Phase Equilib. 1985, 21 (1), $145-155$.

(26) Tohidi, B.; Danesh, A.; Todd, A. C.; Burgass, R. W. HydrateFlee Zone for Synthetic and Real Reservoir Fluids in the Presence of Saline Water. Chem. Eng. Sci. 1997, 52 (19), 3257-3263.

(27) Schicks, J.; Erzinger, J.; Ziemann, M. Raman Spectra of Gas Hydrates-Differences and Analogies to Ice $1 \mathrm{~h}$ and (Gas Saturated) Water. Spectrochim. Acta, Part A 2005, 61 (10), 2399-2403.

(28) Eaves, J.; Loparo, J.; Fecko, C. J.; Roberts, S.; Tokmakoff, A.; Geissler, P. Hydrogen Bonds in Liquid Water are Broken Only Fleetingly. Proc. Natl. Acad. Sci. U. S. A. 2005, 102 (37), 13019-13022.

(29) Pakoulev, A.; Wang, Z.; Dlott, D. D. Vibrational Relaxation and Spectral Evolution Following Ultrafast $\mathrm{OH}$ Stretch Excitation of Water. Chem. Phys. Lett. 2003, 371 (5), 594-600.

(30) Slusher, R; Derr, V. E. Temperature Dependence and Cross Sections of Some Stokes and Anti-Stokes Raman Lines in Ice Ih. Appl. Opt. 1975, 14 (9), 2116-2120.

(31) Braeuer, A. In Situ Spectroscopic Techniques at High Pressure.; Elsevier Science: Amsterdam, 2015.

(32) Qin, J.; Kuhs, W. F. Quantitative Analysis of Gas Hydrates Using Raman Spectroscopy. AIChE J. 2013, 59 (6), 2155-2167.

(33) Garrabos, Y.; Echargui, M.; Marsault-Herail, F. Comparison Between the Density Effects on the Levels of the Raman Spectra of the Fermi Resonance Doublet of the ${ }^{12} \mathrm{C}^{16} \mathrm{O}_{2}$ And ${ }^{13} \mathrm{C}^{16} \mathrm{O}_{2}$ Molecules. J. Chem. Phys. 1989, 91 (10), 5869-5881.

(34) Rosso, K.; Bodnar, R. Microthermometric and Raman Spectroscopic Detection Limits of $\mathrm{CO}_{2}$ in Fluid Inclusions and the Raman Spectroscopic Characterization of $\mathrm{CO}_{2}$. Geochim. Cosmochim. Acta 1995, 59 (19), 3961-3975.

(35) King, M.; Mubarak, A.; Kim, J.; Bott, T. The Mutual Solubilities of Water with Supercritical and Liquid Carbon Dioxides. J. Supercrit. Fluids 1992, 5 (4), 296-302.

(36) Wiebe, R.; Gaddy, V. The Solubility of Carbon Dioxide in Water at Various Temperatures from $12 \mathrm{To} 40^{\circ}$ and at Pressures to $500 \mathrm{~atm}$. Critical Phenomena*. J. Am. Chem. Soc. 1940, 62 (4), 815-817.

(37) Teng, H.; Yamasaki, A.; Chun, M.-K.; Lee, H. Solubility of Liquid $\mathrm{CO}_{2}$ in Water at Temperatures from $278 \mathrm{~K}$ To $293 \mathrm{~K}$ and Pressures from 6.44 MPa To $29.49 \mathrm{MPa}$ and Densities of the Corresponding Aqueous Solutions. J. Chem. Thermodyn. 1997, 29 (11), 1301-1310.

(38) Nakano, S.; Moritoki, M.; Ohgaki, K. High-Pressure Phase Equilibrium and Raman Microprobe Spectroscopic Studies on the $\mathrm{CO}_{2}$ Hydrate System. J. Chem. Eng. Data 1998, 43 (5), 807-810.

(39) Burke, E. A. Raman Microspectrometry of Fluid Inclusions. Lithos 2001, 55 (1), 139-158.

(40) Schuster, J. J.; Will, S.; Leipertz, A.; Braeuer, A. Deconvolution of Raman Spectra for the Quantification of Ternary High-Pressure Phase Equilibria Composed of Carbon Dioxide, Water and Organic Solvent. J. Raman Spectrosc. 2014, 45 (3), 246-252. 